\title{
A Generalized Method for Flat Plates Impact Detection and Location
}

\author{
J.A.Somolinos , R. Morales , C. Morón and A. García
}

Keywords: Impact detection and location, acoustic wave propagation.

\begin{abstract}
In the last years, many analyses from acoustic signal processing have been used for different applications. In most cases, these sensor systems are based on the determination of times of flight for signals from every transducer. This paper presents a flat plate generalization method for impact detection and location over linear links or bars-based structures. The use of three piezoelectric sensors allow to achieve the position and impact time while the use of additional sensors lets cover a larger area of detection and avoid wrong timing difference measurements. An experimental setup and some experimental results are briefly presented.
\end{abstract}

\section{Introduction}

Many analyses from acoustic signal measurement and processing have been carried out during last years. Several experimental applications have been studied using these techniques such as damages detection, failure prevention or some interactive human-machine interfaces (See [1] a.e.), among others. The use of Time Of Flight (TOF) measurements is a common technique from different fields of application such as ultrasonic sensorial systems in mobile robotics, detection of fishing banks and depth of the seabed in navigation assisted tools among others. Old techniques [2] were used for estimating time delay of acoustic signals or more complex algorithms [3] have been used for identification of first time arrival time under the presence of several modes, reflections or other wave distortions. Using the same technique of measuring TOF, the method proposed considers the difference of times of propagation of an acoustic wave when it is produced over a metallic (homogeneous) material based structure, and determines the time instant and the position where the impact or collision has been produced (Details in [4,5 and 6]). This procedure is not interested on detecting structural damages or other events of greater complexity. It results on a very simple, robust and low cost device (details in [7,8]) that is generalized in this paper to a method for impact detection and location over a 2D flat plate. The paper is organized as follows: After a brief introduction, the algorithm for impact over a flat plate detection and location is presented. Next section is devoted to present an experimental setup for validating the proposed algorithms and, finally, some conclusions are stated.

\section{Impact detection}

A flat plate of homogeneous material attending its acoustic properties (ae. metallic ones) is considered. Piezoelectric sensors are located at known positions. Without any loose of generality sensor 1 is placed in the origin of a 2D Cartesian frame. The point where the impact is produced is $P_{I}=\left(\begin{array}{ll}x_{I} & y_{I}\end{array}\right)^{T}$ and the positions of the $n$ sensors with respect to $P_{i}$ with $i=1,2, \cdots, n-1$ are denoted as $P_{i j}=\left(\begin{array}{ll}x_{i j} & y_{i j}\end{array}\right)^{T}$ for $i=1,2, \cdots, n-1, i<j \leq n$, where $\left.P_{11}=\left(\begin{array}{ll}x_{11} & y_{11}\end{array}\right)^{T}=\left(\begin{array}{ll}0 & 0\end{array}\right)^{T}\right)$. If the constant sound velocity, $\mathrm{C}$, is known, the propagation time of the fundamental acoustic wave from $P_{I}$ to each of the $n$ sensors is computed as: 
$t_{i I}=\frac{\left\|P_{i I}\right\|}{C}$ for $i=1,2, \cdots, n$

This time cannot be directly measured because the instant when the impact was produced is not known. An electronic conditioner located near the piezoelectric sensors (see [7] for details) allows convert the acoustic wave received by each of the sensors into a rising edge voltage signal (See Fig. 1a). Measurements based on time differences instead of absolute times are easily achieved from pairs of adapted voltage edge signals (See Fig. 1b obtained from the opt-isolators previous to high speed digital counters).

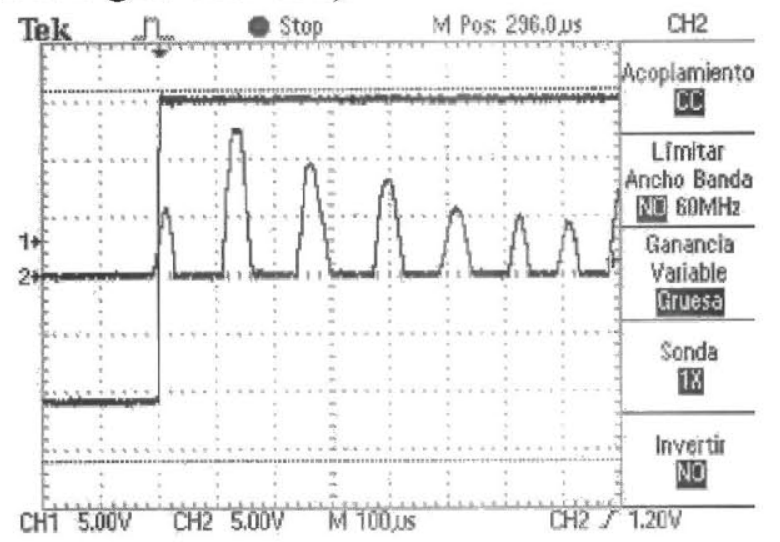

Figure 1a. Conditioned and rising edge signals

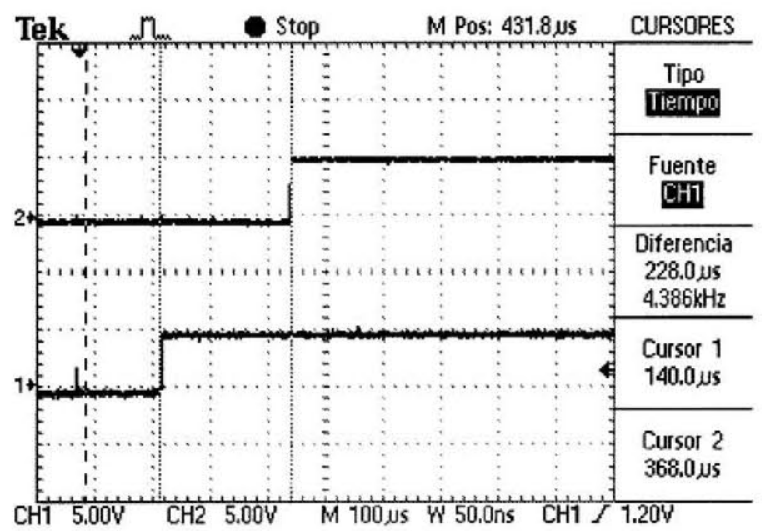

Figure 1b. TTL signals from a pair of sensors

Then, when an impact is detected by any of the sensors, a set of $\left(\begin{array}{l}n \\ 2\end{array}\right)=\frac{n \cdot(n-1)}{2}=N H$ differences of time are obtained. These time differences can be converted to distance differences according with the following expression:

$$
C \cdot \Delta t_{i j}=\left\|P_{i \operatorname{Im} \text { pact }}\right\|-\left\|P_{j \operatorname{Im} \text { pact }}\right\| \text { for } i=1,2, \cdots, n-1, i<j \leq n
$$

where \|\| denotes Cartesian norm. These distances are signed ones. In other words, if the distance between the impact position and $i$-sensor is smaller than the one between the impact position and $j$-sensor, the distance computed, from (4), will be negative, equal to zero if both distances are identical or positive in the other case.

On the other hand, one of the main properties of the hyperbola curve is that differences among each of its points with respect to two points called "focus" are constant and the hyperbola is then composed by two branches: one branch corresponds with the positive differences and the other branch corresponds with negative ones. Fig.2 depicts a family of signed hyperbolas when a pair of sensors (the second one located at $\left.P_{12}=\left(\begin{array}{ll}0 & 100\end{array}\right)^{T}\right)$ determines the both focus of the family of the hyperbolas while the signed distance given by (4) determines the constant of each curve segment (Only positive differences of distances from 0 to 45 equally spaced every 5 units are plotted).

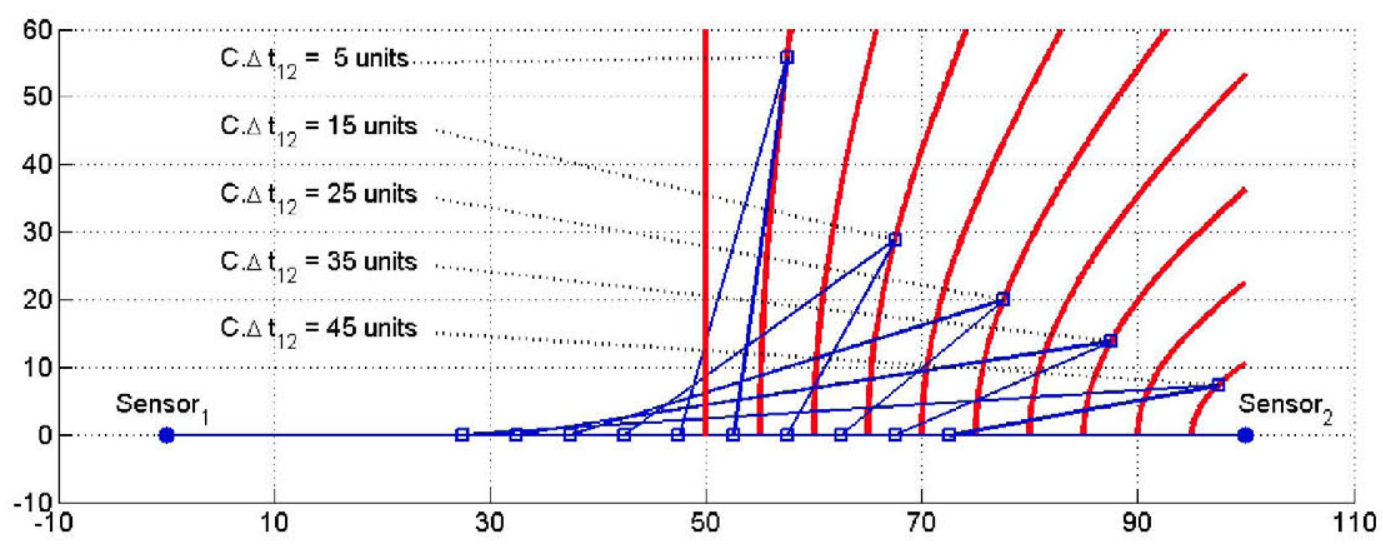

Figure 2. Family of hyperbolas branches for a given pair of sensors 
If $n$ sensors are located on a flat plate, at least all the impacts produced inside the surface bounded by the contour that conform sensors can be detected and $\left(\begin{array}{l}n \\ 2\end{array}\right)=N H$ signed hyperbola branches are obtained. Then, the minimum number of sensors needed for impact location is three, and $\mathrm{NH}$ curves are obtained whose intersection determines the position of impact.

Fig. 3 shows an example of the proposed impact detection procedure. The sensors are located at: $P_{11}=\left(\begin{array}{ll}0 & 0\end{array}\right)^{T}, P_{12}=\left(\begin{array}{ll}100 & 30\end{array}\right)^{T}$ and $P_{13}=\left(\begin{array}{ll}-20 & 100\end{array}\right)^{T}$, and the impact position is simulated at $P_{I}=\left(\begin{array}{ll}28 & 36\end{array}\right)^{T}$ (denoted as a big “+”).

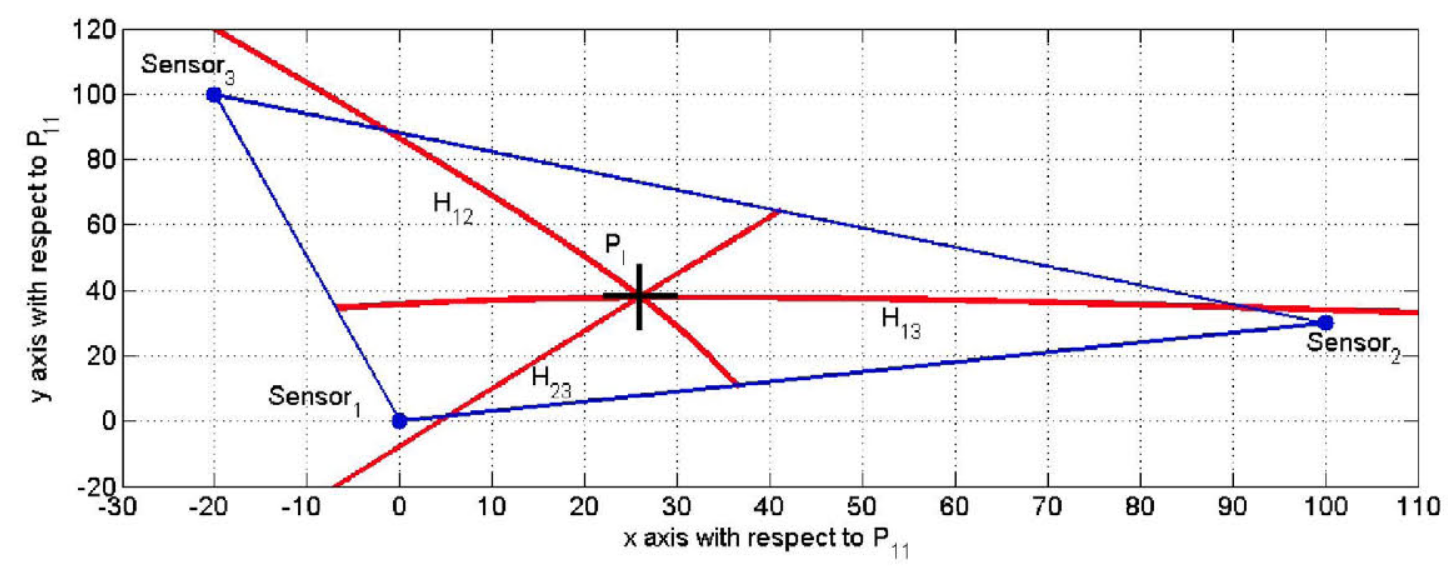

Figure 3. Sensor location, impact point and hyperbolas intersection

From Fig. 3, $\left(\begin{array}{l}3 \\ 2\end{array}\right)=\frac{3 \cdot 2}{2}=3=N H$ hyperbola branches denoted, as $H_{i j}$ for $i=1,2, i<j \leq 3$, have been plotted and every of these hyperbola segments represents the geometric focus of points with the same signed difference of distances (limited by the polygon defined by the sensors). The intersection of these three curves coincides with the point where the impact happened. If we use four sensors instead of three, $\left(\begin{array}{l}4 \\ 2\end{array}\right)=\frac{4 \cdot 3}{2}=6=N H$ hyperbola branches are obtained and their intersection allows to determine the impact position and discard some of the measures of timing differences that may have been wrong.

Once the impact position is known, if it is desired to achieve the time instant when the impact occurred, it can be easily computed from any of the edge signals received by using expressions (1) and (2).

$t_{\operatorname{Im} \text { pact }}=t_{i \operatorname{Im} \text { pact }}-\frac{\left\|P_{i I}\right\|}{C}$ for $i=1,2, \cdots, n$

\section{Experimental Setup and Validation}

Fig. 5 illustrates a general view of an industrial robot Stäubli RX130b with an special knocking tool that has been designed and built for these experiments. This tool is based on a pointer with an internal spring that allows hitting the plate without any damage neither the plate nor the robot. The repeatability of the robot is $\pm 0.05 \mathrm{~mm}$ which ensures a good impact points generation. The steel plate used and the four sensors disposed in a rectangle can be observed in the figure. 
A matrix of impact points were stored into the robot program and all of them are hit with the tool in a random order while the electronic system obtains the time differences which are computed in a PC and converted into distance differences according to eq. (2). After calibrating the system, the errors achieved between the desired impact points and the reconstructed ones are less than the repeatability of the robot which validates the method proposed. (The initial signals in Fig. 1 were captured from one of these experiments).

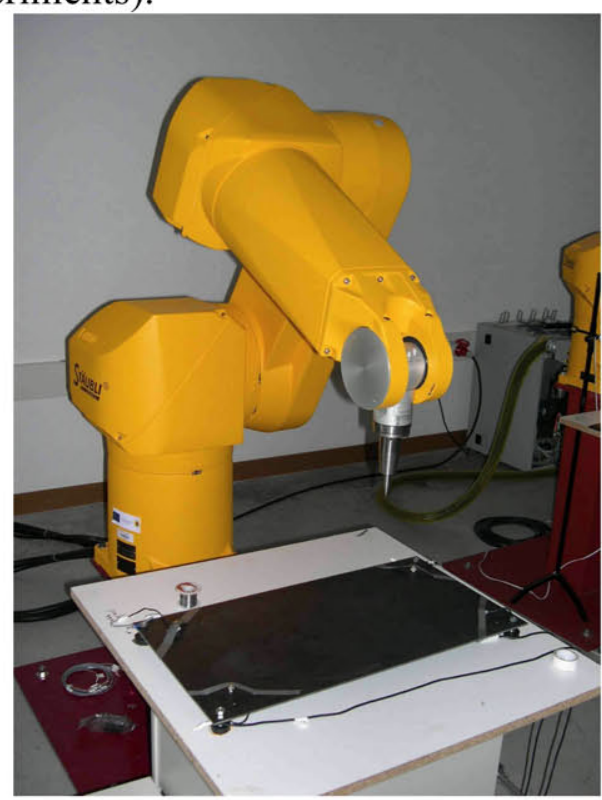

Figure 5. Experimental Setup

\section{Conclusions}

A very simple system which determines the time instant and position of an impact over flat plates with homogeneous acoustic properties has been presented. It is based on measuring the difference of propagation times of the acoustic waves over the plate. According with the geometric convex shape of the plate, a number of sensors are needed for achieving the impact position by calculating the intersection point among a number of signed hyperbola branches. Only three sensors let determine an impact position over a general flat plate. The use of additional sensors allows ensuring the measurements. An experimental setup for validating the system under good repeatability performance has been briefly presented. The proposed method can be applied to more complex metallic non flat surfaces, as those used in shipbuilding. This is the subject of our current research.

\section{Acknowledgements}

This work was partially supported by the JCCM (Spain) under contract no. PAI06-0075 and by the Ministerio de Ciencia e Innovación (Spain) under research grant DPI2011-24113.

\section{References}

[1] Checka N. "A system for tracking and characterising acoustic impacts on large interactive surfaces". MS Thesis, MIT, 2001.

[2] Knapp CH and Carter GC. "The generalized correlation method for estimation of time delay". IEEE Trans. Acoustic, Speech and Signal processing, Vol.24, 1976, pp 320-327.

[3] Ding Y, Reuben RL and Steel JA. "A new method for waveform analysis for estimating AE wave arrival times using wavelet decomposition". NDT \& E International, Vol.37, 2004, pp 279-290.

[4] D. Cortázar, V. Feliu y J.A. Somolinos "Acoustic Impact Detector for Mechanical Structures". Patent ES 2.273.536 B1. (2008) 
[5] J.A. Somolinos, R. Morales. A. Garcia and C. Moron. Piezoelectric Sensors System for Impact Detecting. $8^{\text {th }}$ European Conference on Magnetic Sensors and Actuators. EMSA 2010. Bodrum, (Turkey) July 2010.

[6] Somolinos, J.A., Morales, R., Moron, C. and Garcia, A. "Amorphous piezoresistive and piezoelectric sensors for robotics applications" Physica Status Solidi. Vol. 8. Issue 11-12, pp. 3175-3178. March 2011.

[7] León, C. “Electronic System for Impact Detection and Location”(In Spanish). Master Thesis. E.T.S. Ingenieros Industriales UCLM 03-09-103735

[8] Díaz, J. "Tuning and Application of a New System for Impact Detection And Location" (In Spanish). Master Thesis. E.T.S. Ingenieros Industriales UCLM 05-09-200512 
Materials and Applications for Sensors and Transducers II

10.4028/www.scientific.net/KEM.543

A Generalized Method for Flat Plates Impact Detection and Location

10.4028/www.scientific.net/KEM.543.171 